established. Other possible indirect mechanisms of clozapine predisposition to infection, particularly aspiration pneumonia, include sialorrhoea ${ }^{2}$ and impairment of swallowing function with oesophageal dilatation and hypomotility. ${ }^{3}$ However, less is known about whether clozapine has more direct pro-inflammatory effects. It has been argued that clozapine has a direct influence on different cytokines resembling an inflammatory reaction and that infection or inflammation could induce bioactivation of clozapine into its nitrenium ion. ${ }^{4}$ The latter can exert a toxic reaction that induces apoptosis and gives rise to elevated cytokine levels. ${ }^{4}$ However, these arguments are still awaiting robust research assessment.

Regardless of the cause of infection, a number of reports ${ }^{4-6}$ showed that infection leads to a rise of toxic levels in serum clozapine and its metabolites. This is likely to be mediated by cytokine suppression of cytochrome P450 1A2 (CYP1A2), the main hepatic microsomal system involved in clozapine metabolism; CYP1A2 is also involved in the metabolism of a number of antibiotics in common use for treating infections. This enhances further potentials for clozapine toxicity.

We wholeheartedly agree with Taylor et al that any bronchial infection (or indeed other infections, including wound infection) should attract immediate attention. Clinicians should bear in mind that both the infection and the drug treatment of the infection (through drug-drug interactions at CYP1A2) can lead to very high and toxic levels of serum clozapine that may lead to more adversities. In such circumstances we recommend monitoring for signs of clozapine intoxication (e.g. speech dysfluency, myoclonus and increased sedation), obtaining clozapine levels, considering significant clozapine dose reduction and working closely with physical health physicians in deciding about the most appropriate antimicrobial therapy and required supportive measures.

1 Taylor DM, Douglas-Hall P, Olofinjana B, Whiskey E, Thomas A. Reasons for discontinuing clozapine: matched, case-control comparison with risperidone long-acting injection. Br J Psychiatry 2009; 194: 165-7.

2 Hinkes R, Quesada TV, Currier MB, Gonzalez-Blanco M. Aspiration pneumonia possibly secondary to clozapine-induced sialorrhea. J Clin Pharmacol 1996; 16: $462-3$.

3 Maddalena AS, Fox M, Hofmann M, Hock C. Esophageal dysfunction on psychotropic medication. A case report and literature review. Pharmacopsychiatry 2004; 37: 134-8.

4 Haack MJ, Bak ML, Beurskens R, Maes M, Stolk LM, Delespaul PA. Toxic rise of clozapine plasma concentrations in relation to inflammation. Euro Neuropsychopharmacol 2003; 13: 381-5

5 Raaska K, Raitasuo V, Arstila M, Neuvonen PJ. Bacterial pneumonia can increase serum concentration of clozapine. Euro J Clin Pharmacol 2002; 58: 321-2.

6 De Leon J, Diaz FJ. Serious respiratory infections can increase clozapine levels and contribute to side effects: a case report. Prog Neuropsychopharmacol Biol Psychiatry 2003; 27: 1059-63.

Nasser Abdelmawla, Teal Psychiatric Intensive Care Unit, Northamptonshire Healthcare NHS Trust, 77 London Road, Kettering NN15 7PW. Email:

nasser.abdelmawla@nht.northants.nhs.uk; Magdi I. Ahmed, Northamptonshire Healthcare NHS Trust, Kettering, UK

doi: 10.1192/bjp.194.5.468a

\section{Dual diagnosis quandries}

Morgan et al have made a useful contribution in the area of intellectual disability/mental illness dual diagnosis. ${ }^{1}$ However, this study, like most in this area, is flawed by inadequate definition of terms. 'Intellectual disability', the current phrase of fashion for this population, is unsatisfactory because many individuals in the higher IQ ranges are not disabled. The American Association on
Mental Retardation (now AAIDD) definition, probably the most widely used definition, is cited. It gets around the disability issue by requiring that individuals with intellectual disability must also have 'limitations in adaptive behaviours and skills'. This confounds the intellectual disability and mental illness categories, as such limitations may well be due to mental illness. Perhaps a better term for studies to use would be 'intellectual impairment', which, like visual impairment, does not necessarily imply disability; then, all individuals in certain IQ ranges could be included. As it is, a certain proportion of individuals without mental illness are excluded by the definition. This may inflate the prevalence rates.

Additionally, there is a problem in lumping together all ranges of intellectual disability. As Morgan et al note, mental illness, particularly schizophrenia, is more likely to be diagnosed in the borderline group and pervasive developmental disorder is more likely to be diagnosed in the severe/profound group. Rather than a true reflection of incidence, this may reflect a nosological bias. A strict definition of schizophrenia is difficult to apply to a non-verbal person. Historically, pervasive developmental disorder and schizophrenia have sometimes been used interchangeably in apparently disturbed and non-verbal individuals; however, since the 1990s, at least in the USA, there has been a massive shift towards the diagnosis of pervasive developmental disorder subcategories such as autism and Asperger syndrome. The diagnosis of schizophrenia has an additional stigma which some families find unacceptable. The authors found some trends distinguishing individuals with dual diagnosis from those with intellectual disability alone. Some of these trends also distinguished borderline from other levels of intellectual disability (e.g. fewer genetic causes, less Down syndrome, less epilepsy). To distinguish dual diagnosis from intellectual disability alone, results should probably be controlled for IQ level.

Morgan et al have considered patients with dual diagnosis to have more severe mental illness than other patients with mental illness as indicated by number of hospitalisations, length of hospitalisations, etc. Perhaps this just indicates that treatment and placement options for these patients are poorer. Future studies need to be done to clarify the unique aspects of this population.

1 Morgan VA, Leonard H, Bourke J, Jablensky A. Intellectual disability co-occurring with schizophrenia and other psychiatric illness: populationbased study. Br J Psychiatry 2008; 193: 364-72.

Patricia E. Hogan, Family Guidance Center, St. Joseph, MO, USA

Email: drpathogan@hotmail.com

doi: 10.1192/bjp.194.5.469

Authors' reply: We thank Patricia Hogan for her comments challenging current definitions of intellectual disability and highlighting the difficulty of accurate assessment of psychotic illness in individuals with intellectual disability. With respect to the former, we note the importance of applying standard definitions and nomenclature in the study of the epidemiology of dual diagnosis. The criteria used to define intellectual disability affect prevalence rates and the use of IQ criteria alone rather than the dual criteria of IQ and adaptive behaviours will have a marked impact on rates. ${ }^{1}$ We employed the American Association on Mental Retardation dual criteria in our study. The use of dual criteria is the most common approach across services and in research, and is consistent with DSM-IV and ICD-10 definitions. As the American Association on Mental Retardation criteria are the basis of service eligibility in Western Australia, their use ensures a thorough assessment of individuals on the intellectual disability register and greater confidence that cases have been 